\title{
Análise de Sentimento pela ótica da abordagem multimodal
}

\author{
Luciana Kraemer da Silva ${ }^{1}$, Maria Lúcia Kroeff Barbosa ${ }^{1}$, \\ Robson Pandolfi ${ }^{2}$, Sílvio César Cazella ${ }^{1,3}$ \\ ${ }^{1}$ Programa de Pós-Graduação em Informática na Educação - UFRGS \\ ${ }^{2}$ Programa de Pós-Graduação em Computação Aplicada - UNISINOS \\ ${ }^{3}$ Programa de Pós-Graduação em Ensino na Saúde - UFCSPA \\ luciana.kraemer@gmail.com, malukroeff@yahoo.com.br, \\ robson@pandolfi.jor.br, silvio.ufcspa@gmail.com
}

Resumo. A Análise de Sentimento é um campo de estudo importante para a Computação Afetiva e para a compreensão dos processos cognitivos em geral. Dois aspectos embasam essa constatação: a web ter se tornado uma arena pública para difusão de opiniões; e a comunicação, desde a mais tenra idade, ser expressada a partir de múltiplas linguagens. Este artigo apresenta um estudo sobre análise de sentimento via web com base em objetos multimodais e multilíngues. Com base em pressupostos teóricos sobre análise de sentimento e multimodalidade com abordagem multilíngue, pretende-se explorar aspectos relevantes dos estudos em questão, bem como comparar atuais técnicas desenvolvidas sobre esse tema.

Palavras-Chave: análise de sentimentos, abordagem multimodal, computação afetiva, mineração de opinião

\section{Sentiment analysis by optical of multimodal approaches}

\begin{abstract}
Sentiment Analysis is an important field of study for Affective Computing and also for a better understanding of general cognitive processes. Two aspects confirm this observation: the web has become a public arena for diffusion of opinion, and communication, since humans from an early age, express themselves in multiple languages. This paper presents a study on the analysis of sentiment on the Internet from a multimodal perspective. Based on theoretical assumptions about Sentiment Analysis and multimodality, we intend to explore relevant aspects of the studies in question, as well as compare different methods and approaches of analysis.
\end{abstract}

Keywords: sentiment analysis, multimodal approach, affective computing, opinion mining

\section{Introdução}

A análise de sentimentos via web é um campo em expansão. Segundo Pang e Lee [2008], a expressão começa a aparecer como questão de pesquisa em meados dos anos 2001. A área se firmou nos anos seguintes, em artigos acadêmicos apresentados em conferências acadêmicas que tinham como foco computação e linguística, mais precisamente, em centros de estudos associados ao processamento da linguagem natural (NLP). Nos últimos 
anos, centenas de artigos foram escritos sobre esse tema, e muitas startups que medem opinião sobre dados reais têm surgido para propor métodos de análise de sentimento, além de pacotes estatísticos que incluem módulos de análise de sentimento [Araújo,2016].

$\mathrm{O}$ crescente interesse se deve à ascensão da web como arena pública para compartilhar opiniões e sentimentos acerca de todas as áreas de nossas vidas. Como nos diz Cambria et al. [2013], a principal fonte de informação sobre qualquer área de interesse dos indivíduos são os amigos e as publicações especializadas. Hoje, a web disponibiliza toda a sorte de ferramentas (representadas especialmente nas mídias sociais) para criar e compartilhar opiniões. Mesmo que se tratem de microtextos (Twitter, Facebook, fóruns de mensagens, $b \log s$, fóruns de usuários), estes espaços representam uma mina de ouro para empresas e indivíduos que desejam monitorar reputações e obter feedback em tempo útil sobre ações e produtos - incluindo-se, aí, as instituições de ensino, em virtude do crescente aumento de cursos online. Para Benevenuto et al. [2015], as redes sociais são a criação de uma revolução digital. Elas permitem a expressão e difusão das emoções e opiniões através da rede, uma vez que são locais onde as pessoas discutem sobre tudo expressando opiniões políticas, religiosas ou mesmo sobre marcas, produtos e serviços. Os autores ainda colocam que as opiniões nas redes sociais, se devidamente coletadas e analisadas, permitem não só compreender e explicar diversos fenômenos sociais complexos, mas também prevê-los. No contexto da educação, a avaliação dos sentimentos dos alunos oportuniza um material valioso para que professores e tutores conduzam melhor suas aulas, tanto no que se refere à elaboração do conteúdo, como na consequente disponibilização online.

Benevenuto et al [2015], Reis [2015] e Malheiros e Lima [2013] apontam que existe um grande número de estratégias utilizadas para aferir sentimentos, como escalas psicométricas adaptadas para modelos computacionais, dicionários léxicos, t écnicas de aprendizado de máquina e processamento de linguagem natural. Mas grande parte desses recursos estão disponibilizados somente na língua inglesa, o que limita seu uso, sendo que alguns esforços já têm sido realizados para o desenvolvimento de técnicas de análise de sentimentos em outros idiomas.

Este artigo está estruturado em seis seções, incluindo introdução e conclusão. Na seção 2, as referências teóricas que embasam o conceito de análise de sentimento como um campo em desenvolvimento da Computação Afetiva, e seu entendimento a partir de alguns grupos e pesquisadores especialistas na área. A seção 3 aborda a teoria da multimodalidade e sua relevância para a análise de sentimentos no meio digital, além da compreensão dos conceitos de multimodalidade, a partir do campo da Educação. Na seção 4, serão descritos alguns trabalhos relacionados. $\mathrm{Na}$ seção 5, são apresentadas e comparadas algumas das principais metodologias utilizadas para análise. Por fim, na seção 6, serão realizadas as considerações finais.

\section{A análise de sentimentos e a Computação Afetiva}

Para Cambria et al. [2012], emoções são complexos estados de sentimentos que resultam em reações físicas e psicológicas, pensamento e comportamento e é um dos temas mais difíceis e abertos da psicologia, especialmente pelo aspecto ambíguo da língua. A linguagem é considerada uma parte vital da experiência emocional, e tem forte componente cultural. O autor utiliza como o exemplo o significado de palavras como amor, raiva ou medo, que possuem diferentes interpretações dependendo do contexto. Além disso, 
algumas palavras nem sempre encontram equivalência em culturas diferentes das que foram criadas. Poderíamos citar como exemplo na língua portuguesa, a palavra saudade.

O estudo de opiniões e emoções quer compreender de que maneira os sentimentos são expressos a partir de opiniões negativas e positivas sobre determinado assunto. As técnicas envolvem pesquisa de campo e questionários [Becker e Tumitan, 2013]. No entanto, esse tipo de abordagem costuma ter custos elevados e, via de regra, limita-se a amostras reduzidas. A ascensão das mídias sociais e a multiplicação das possibilidades de expressão de opiniões e sentimentos na web mudaram esse cenário. Conforme Pang e Lee [2008], os termos análise de sentimento e mineração de opinião começam a surgir nos estudos da computação no início do século 21 , e o seu início relacionado ao desenvolvimento dos métodos de aprendizagem de máquina e de processamento da linguagem natural.

Uma opinião é composta basicamente por dois elementos: um alvo e um sentimento sobre esse alvo - que pode ser uma pessoa, um assunto, um produto, uma marca etc. O sentimento, por sua vez, é uma atitude ou emoção de alguém a respeito do seu alvo. A mineração e a classificação de opiniões trouxeram desafios significativos. Em primeiro lugar, o volume de dados gerados diariamente exige o domínio de métodos, técnicas e ferramentas para processar esse tipo de conteúdo. Determinar a polaridade de uma opinião ou sentimento também exige, na maior parte dos casos, uma análise do contexto em que a mensagem está inserida [Becker e Tumitan, 2013]. Além disso, o campo requer um forte entendimento da linguagem, tanto ao que está explícito, como ao implícito - exigindo, portanto, o reconhecimento de regras sintáticas e semânticas. Este segundo aspecto - isto é, a preocupação de fazer uma distinção entre a linguagem objetiva, das subjetividades implicadas nos textos - tem sido um objetivo presente desde os primeiros trabalhos publicados na área.

Vários artigos têm sido escritos sobre as tarefas possíveis a serem desempenhadas pela análise de sentimento. Para Becker e Tumitan [2013], a maior parte dos trabalhos se concentra no desenvolvimento de técnicas de detecção e sumarização automática de opiniões sobre produtos ou serviços. Dentre as mais utilizadas, destacam-se o reconhecimento de emoções, a detecção de polaridade e a fusão multimodal. A última está relacionada ao fato da expressão audiovisual estar cada vez mais presente na nossa sociedade, uma vez que a webcam se integrou aos dispositivos de usuário final (smarfones, tablets, notebooks). Isso possibilita uma quantidade crescente de informações afetivas em formato multimodal despejadas nas redes sociais. Por fusão multimodal entende-se a integração de todas as modalidades individuais em uma representação única, combinada. A partir da fusão é possibilitado o reconhecimento de voz para texto, a exploração adicional de informação acústica, expressão facial, análise de movimento do corpo, etc.

\section{Multimodalidade e Multiletramento}

Os conceitos multimodais que podem ser utilizados em análises de textos produzidos a partir de diversos modos de linguagem são desenvolvidos a partir da teoria da multimodalidade [Kress e Van Leeuwen, 2001], não se pensando mais isoladamente em cada um deles. Essa visão se apoia no argumento de que a comunicação humana é fundamentalmente multimodal, uma vez que os modos semióticos não funcionam separadamente, mas em uma interação, onde todos realizam os significados que fazem parte de seu potencial semiótico [Kress, 2010]. Sendo assim, importante considerar, nesse 
olhar sobre o uso de linguagens, que os modos (ou recursos semióticos) funcionam em conjunto, e cada um deles contribui de acordo com a sua capacidade de fazer significados.

Para Serafini [2013], a linguagem multimodal se traduz como as formas de recorrer a diferentes recursos semióticos para articular, representar e interpretar signos. Algumas modalidades apresentam duas dimensões (por exemplo, a fotografia e a pintura), e outras três (por exemplo, a escultura). Todas mostram aspectos materiais, psicológicos, tecnológicos, socioculturais, sendo que um dos conceitos chaves dos múltiplos modos é que nenhuma pode expressar sozinha o conceito ou significado. Conforme Cope e Kalantizis [2000], que juntamente com outros autores lançaram a base para uma pedagogia do Multiletramento (The New London Group - NLG) para uma reforma no ensino escolar, os processos de significação ocorrem a partir de diversas linguagens combinadas, sendo o código escrito um dos tantos a concorrer com o sistema de signos presentes nos novos ambientes de comunicação, especialmente a web. O sentido de letramento, objetivo primordial da escola, não se restringe à alfabetização. O NLG também discutiu a centralidade do inglês no processo de letramento. Mesmo admitindo a supremacia da língua inglesa, os pesquisadores entenderam que o crescimento da multiculturalidade propiciado pela globalização, a partir dos fluxos migratórios e pela conectividade revelaram uma diversidade de sotaques e culturas. Negociar com estas diferenças linguísticas é uma habilidade necessária, pois afeta a todas as áreas da vida, como relações pessoais, de trabalho, políticas, e comunidade em geral.

Vian Jr. [2011] entende que a chegada das novas tecnologias da informação e da comunicação possibilitaram novos modos de comunicação, novos gêneros e suportes. Esses são os verdadeiros agentes de mudanças sociais, visto que as comunidades sofrem alterações devido à assimilação dessas novas práticas. Observando -se os novos recursos multimodais, pode-se notar a forma como a dinâmica social foi alterada em função das novas redes sociais e os gêneros exigidos em tais redes, como, por exemplo, Twitter ou Facebook.

Tratando-se especificamente da multimodalidade empregada na análise de sentimentos, percebe-se que essas redes sociais online tornaram-se uma importante plataforma de comunicação que reúne muitas informações, entre elas opiniões e sentimentos expressos por seus usuários em simples conversas ou mensagens. A quantidade de usuários ativos e o volume de dados criados diariamente nessas redes é impressionante. Porém, a dificuldade está em extrair opiniões e informações que sejam de fato relevantes, e esta dificuldade gera um problema multidisciplinar na computação, envolvendo a mineração de dados, o processamento de linguagem natural e a aprendizagem de máquina. A fim de tornar os dados textuais qualitativos efetivamente, a quantificação de "o que as pessoas pensam" torna-se um passo obrigatório, desafiador e de interesse de diversas comunidades científicas [Benevenuto, 2015].

\section{Trabalhos Relacionados}

Existem inúmeras pesquisas na área de análise de sentimentos. O crescimento do conteúdo web disponibilizado em outros idiomas além do inglês, e a ampliação das técnicas impulsionaram o desenvolvimento de trabalhos que têm como objetivo explorar a análise de textos contendo opiniões ou sentimentos - independentemente do idioma no qual estão 
escritos. Surgem ainda, os que apresentam proposta de análise multimodal de sentimento e opinião, conceituando multimodal como evidências extraídas de áudio, vídeo e texto.

Grande parte dos trabalhos acessados na revisão teórica realizada para este estudo traz também um comparativo entre as ferramentas de análise automática e as de análise humana, a fim de detectar o grau de eficiência e acurácia entre os métodos. As técnicas de aprendizado de máquina aplicadas à análise de sentimento se dividem entre abordagens supervisionadas e não supervisionadas [Benevenuto et al, 2015]. Nesta última, destacamse ferramentas que utilizam a abordagem léxica - baseadas em dicionários léxicos de sentimento, um dicionário que, em vez de trazer o significado de uma palavra, traz uma classificação que vai de -1 a 1 , onde -1 representa o valor sentimental mais negativo possível e 1 , o mais positivo.

Entre as ferramentas que se utilizam da abordagem não supervisionada, estão: SenticNet, que um conjunto de ferramentas e técnicas para análise de sentimentos que combina raciocínio de senso-comum, psicologia, linguística e aprendizagem de máquina; SentiWord, baseado no dicionário léxico WordNet, que agrupa adjetivos, verbos e outras classes gramaticais em conjuntos (synset) em uma abordagem semi-supervisionada; Lexicon Sentiment $140^{1}$, também conhecido como "Twitter Sentiment", que permite que se descubra os sentimentos relacionados a uma marca, produto ou tópico no Twitter; e Vader (Valence Aware Dictionary for sEntiment Reasoning) ${ }^{2}$, ferramenta de análise de sentimentos desenvolvida com foco em avaliar mensagens no contexto do Twitter e outras mídias sociais online que não requer treinamento.

Outros métodos combinam as polaridades definidas pelos dicionários léxicos com outras heurísticas e processamentos baseados em pistas que vão além da polaridade das palavras, baseando-se em outras pistas gramaticais, como pontuação - o número de exclamações no final de uma frase, por exemplo - e capitalização - uma frase escrita inteiramente em letras maiúsculas costuma indicar uma intensidade maior de um sentimento [Benevenuto et al, 2015]. O Vader é uma das ferramentas que utilizam essa abordagem. Na tabela 1, são listados alguns dos métodos comuns de análise automática, assim como suas características.

Tabela 1. Metodologias, abordagens e técnicas de análise de sentimentos

\begin{tabular}{|l|l|}
\hline \multirow{2}{*}{$\begin{array}{l}\text { Dados } \\
\text { rotulados }\end{array}$} & $\begin{array}{l}\text { Trata-se da associação de polaridades a palavras e sentenças. Para que o modelo seja eficaz } \\
\text { é preciso, antes, rotular uma amostragem significativa do domínio do problema para treino } \\
\text { ou testes. Uma das técnicas utilizadas é o Distant Supervisor, que utiliza características } \\
\text { preexistentes no texto para rotulação automática. Também é possível definir a polaridade } \\
\text { por meio de emoticons - um =) poderia ser classificado como positivo. Um dos problemas } \\
\text { dessa abordagem é a subjetividade para rotular sentenças. }\end{array}$ \\
\hline $\begin{array}{l}\text { Definição de } \\
\text { features }\end{array}$ & $\begin{array}{l}\text { Um conjunto de features é composto pelas próprias palavras presentes no texto. Na } \\
\text { representação conhecida como bag of words, cada sentença é representada por uma lista } \\
\text { de termos associados a 0's e 1's, que indicam, respectivamente, a presença ou ausência de } \\
\text { uma palavra. Nas sentenças The restaurant is good! e The restaurant is not good!, a feature } \\
\text { "good" provavelmente estará relacionada a uma conotação positiva. No entanto, na } \\
\text { segunda frase, a existência do "not" muda a polaridade da frase. }\end{array}$ \\
\hline
\end{tabular}

\footnotetext{
1

http://help.sentiment140.com/home

https://pypi.python.org/pypi/vaderSentiment
}

V. $15 \mathrm{~N}^{\mathrm{o}} 1$, julho, 2017 


\begin{tabular}{|l|l|}
\hline \multirow{2}{*}{$\begin{array}{l}\text { Léxico de } \\
\text { sentimentos }\end{array}$} & $\begin{array}{l}\text { Atualmente, é uma das abordagens mais eficazes. O léxico de sentimentos utiliza grandes } \\
\text { dicionários de termos (veja no item a seguir). Nele, cada termo é associado a um } \\
\text { sentimento. Quando o método recebe uma sentença de entrada, inicia-se o processamento } \\
\text { de linguagem natural - quando as mensagens são quebradas em peças gramaticais, tarefa } \\
\text { conhecida como POS (Part-of-Speech), que pode ser realizada por uma série de bibliotecas } \\
\text { em diversas linguagens de programação - e a pesquisa no léxico dos termos que formam a } \\
\text { frase, permitindo que o método infira a polaridade ou sentimento implícito na sentença. }\end{array}$ \\
\hline \multirow{5}{*}{$\begin{array}{l}\text { Construção de } \\
\text { dicionários }\end{array}$} & $\begin{array}{l}\text { Uma das formas mais confiáveis para criar dicionários léxicos é a validação manual de } \\
\text { "significados sentimentais" para palavras. No entanto, também é a mais trabalhosa, } \\
\text { levando-se em conta o volume textual produzido na web e a informalidade da linguagem } \\
\text { nas mídias sociais. Há, no entanto, ferramentas que podem ajudar nessa tarefa, como } \\
\text { LIWC, General Inquirer (GI), Opinion Lexicon, Anew, Senticnet, Sentiment 140, Vader e } \\
\text { Sentiword. }\end{array}$ \\
\hline SVM & $\begin{array}{l}\text { SVM (Support Vectors Machine, ou Máquinas de Vetores de Suporte Vetores, em livre } \\
\text { tradução) é o termo utilizado para um conjunto de métodos de aprendizado supervisionado } \\
\text { que difere de outros classificadores por não utilizar uma separação linear entre os dados, } \\
\text { mas utilizar funções que, quando multiplicadas ao conjunto de dados, permitem a melhor } \\
\text { separação entre as diferentes entradas. Para Lorena and De Carvalho [2008], o SVM é um } \\
\text { dos principais métodos de aprendizado de máquina e é aplicado a domínios como } \\
\text { categorização de textos, análise de imagens e bioinformática. }\end{array}$ \\
\hline
\end{tabular}

Fonte: Adaptado de Benevenuto et al, 2015.

\section{Materiais e Métodos}

O estudo apresentado neste artigo constitui-se em pesquisa exploratória, uma vez que pesquisas com objetivo exploratório são desenvolvidas para proporcionar visão geral, de tipo aproximativo, acerca de determinado fato e geralmente constituem a primeira et apa para uma investigação mais ampla. Por conseguinte, com o intuito de elaborar uma comparação que contemple as mais recentes ferramentas para análise de sentimentos, as buscas foram realizadas com base nos seguintes critérios:

$\checkmark$ Métodos de análise multimodal de sentimentos, onde a multimodalidade implica no uso de várias mídias além do textual, tais como áudio e vídeo, para aumentar a precisão da classificação do sentimento pelos analisadores de conteúdo emocional.

$\checkmark$ Métodos de análise de sentimentos que priorizam uma abordagem multilíngue, favorecendo que outros idiomas, não apenas o inglês, possam ser minerados.

Com base no descrito, optou-se por comparar o que mais se alinha ao conceito multimodal e elencar as principais ferramentas que se utilizam de diferentes métodos de análise de sentimento de máquina. Tais resultados seguem descritos na próxima seção.

\section{Resultados}

No que se refere à multimodalidade, Cambria [2016], entende que dois tipos de técnicas de fusão têm sido utilizadas para melhorar a confiabilidade e reconhecimento a partir da análise multimodal de sentimento, a feature-level fusion (fusão de nível de recurso) e a decision-level fusion (fusão de nível de decisão). Dentre os estudos citados pelo autor que utilizaram análise multimodal, estão os de Stephan Raaijmakers et al. [2008] e Louis- 
Philippe Morency et al. [2011]. O último identificou palavras polarizadas, sorrisos, olhares, pausas e tons de voz como características relevantes para emoção, porém a análise textual teria sido feita manualmente. O destaque fica para o recente estudo de Soujanya Poria et al. [2016] que propôs uma nova metodologia para análise de sentimento multimodal com grau de precisão de aproximadamente $80 \%$. Para tanto, foi efetuada a integração da informação cognitiva e afetiva a partir das estratégias feature level fusion e decision level fusion. De acordo com Poria et al. [2016], uma série de estudos favorecem o nível de decisão de fusão porque os erros de diferentes classificadores não são correlacionados e a metodologia independente do recurso. Para fazer a análise multimodal de sentimento foram utilizadas as seguintes bases de dados:

$\checkmark$ A ISEAR (Survey of Emotion Antecedents and Reactions), base de dados [Scherer, 1996]. A pesquisa foi conduzida nos anos 90 em 37 países e 3.000 respondentes. Os respondentes foram instruídos a descrever uma situação ou evento que tivessem sentido uma emoção particular no formato de um texto pequeno. Várias características que estão nas bases de SenticNet e outras ferramentas foram extraídas de ISEAR.

$\checkmark$ Para construir o modelo para reconhecimento de emoções a partir de expressões faciais, foi usado o $\mathrm{CK}++$ é um conjunto de dados abrangente que consiste de imagens do comportamento facial de 210 adultos. As imagens sequências foram gravadas usando dois hardwares sincronizados, câmeras Panasonic AG-7500. Os participantes tinham entre 18-50 anos 81\% euroamericanos, $13 \%$ dos afro-americanos, e $6 \%$ de outros grupos étnicos; $69 \%$ eram do sexo feminino.

$\checkmark$ O eNTERFACE é um banco de dados em que estão gravados 42 indivíduos de 14 nacionalidades. Eles foram submetidos a escuta de seis contos sucessivos, cada um deles provocando uma emoção particular (seis emoções básicas de Ekman foram utilizadas). Eles foram instruídos pelo experimentador para reagir a cada uma das seis situações (histórias).

$\checkmark$ E ainda SenticNet, ConceptNet, EmoSenticNet, EmoSenticSpace.

A partir desta fusão, e criação de vetores para análise, os autores comprovaram que obtiveram o grau de precisão de $87,95 \%$, superando em mais de $10 \%$ os experimentos demonstrados no state of art realizado. Ou ainda, apresentando uma redução de $56 \%$ na taxa de erro demonstradas nos demais sistemas. Já no que se refere ao iFeel, ainda quando o sistema estava em 1.0, Reis et al. [2015] identificaram que a ferramenta foi abrangente, levando em conta o percentual de mensagem em que cada método conseguiu detectar algum sentimento, positivo ou negativo.

Neste trabalho, os métodos SenticNet, Sentiword e Sentiment140 Lexicon alcançaram as 3 mais altas abrangências, mostrando vantagens em relação a todos os idiomas. Mas em relação à acurácia, Vader, SentiStrength e Emoticons se saíram melhores. Já em Reis [2015], onde foram analisados 21 métodos, o Sentistrength mostrou ser o mais acurado para a tarefa de análise de sentimento automático, mas identificou-se que o SOCAL, Umigon e Vader, são métodos que mostraram estar habilitados com boa acurácia, portanto, revelando performance satisfatória em diferentes bancos de dados, considerando o MacroF1 e abrangência. 


\section{Considerações Finais}

A web se torna cada vez mais um campo para exposições de ideias e contínua tomada de decisão. A premissa vale para todos os aspectos da vida pública e privada, com impacto direto no desenvolvimento social das comunidades. A multimodalidade é outro fator cada vez mais presente na cultura. Importante salientar, trata-se de um conceito pertinente para uma discussão mais ampla, em relação à ideia de que, na sociedade atual, graças à tecnologia, a comunicação é configurada cada vez mais por textos digitais e por supo rtes audiovisuais. Para os autores citados, especialmente Cambria [2016], a combinação de teorias científicas de emoção, com técnicas computacionais para analisar sentimentos em linguagem natural permite a construção de caminhos para lidar com conhecimento semântico, fazer analogias e aprender mais sobre conhecimento afetivo. Para detectar, perceber e sentir as emoções. Ao mesmo tempo, faz-se necessário testar os diferentes métodos que surgem, e validá-los para diferentes idiomas, não apenas no inglês. Analisar a precisão, a abrangência e fazer comparações entre as ferramentas que estão surgindo é algo que se torna cada vez mais necessário em diferentes áreas da sociedade - e um dos desafios para este campo de pesquisa.

\section{Referências}

ARAÚJO, M. et al."iFeel 2.0: A Multilingual Benchmarking System for Sentence Level Sentiment Analysis", 2016. Disponível em:

<http://homepages.dcc.ufmg.br/ fabricio/download/icwsm2016-ifeel2.pdf>. Acesso em junho de 2016.

, M. et al.“An Evaluation of Machine Translation for Multilingual Sentence level Sentiment Analysis”, 2016. Disponível em:

$<$ https://homepages.dcc.ufmg.br/ fabricio/download/sac2016-translation.pdf $>$. Acesso em junho de 2016.

, M. et al."iFeel: A System that Compares and Combines Sentiment Analysis Methods". In Proceedings of the World Wide Web Conference (WWW' 14). Seoul, Korea. April 2014. Disponível em:

<http://homepages.dcc.ufmg.br/ fabricio/download/de03-araujo.pdf>. Acesso em julho de 2016.

, M. et al.“Métodos para Análise de Sentimentos no Twitter”. In: Brazilian Symposium on Multimedia and the Web (Webmedia), 2013, Salvador. Disponível em: <http://homepages.dcc.ufmg.br/ fabricio/download/webmedia13.pdf >. Acesso em julho de 2016.

BECKER, K.; \& TUMITAN, D.“Introdução à Mineração de Opiniões: Conceitos, Aplicações e Desafios”. Simpósio Brasileiro de Banco de Dados, 2013.

BENEVENUTO, F.; RIBEIRO, F.; ARAÚJO, M.“Métodos para Análise de Sentimentos em Mídias Sociais". In: Webmedia2015(minicurso). Disponível em: <http://homepages.dcc.ufmg.br/ fabricio/download/webmedia-short-course.pdf >. Acesso em julho de 2016.

CAMBRIA, E. “Affective computing and sentiment analysis."IEEE Intelligent Systems 31(2), pp.102-107(2016). Disponível em: <http://sentic.net/affective-computing-andsentiment-analysis.pdf > . Acesso em maio de 2016. 
CAMBRIA, E. et al."New Avenues in Opinion Mining and Sentiment Analysis". IEEE Intelligent Systems, v.28, n.2, p.15-21, mar.2013. Disponível em: <http://sentic.net/new-avenues-in-opinion-mining-and-sentiment-analysis.pdf> . Acesso em julho de 2016.

CAMBRIA, E.; LIVINGSTONE, A.; HUSSAIN, A. "The Hourglass of Emotions" Cognitive Behavioral Systems, LNCS7403, 2012, pp.144-157. Disponível em: <http://sentic.net/hourglass-of-emotions.pdf>. Acesso em agosto de 2016.

CARVALHO, J. "Uma estratégia estatística e evolutiva para mineração de opiniões em tweets”. 2014.80p.Dissertação de Mestrado. PPG em Computação da Universidade Federal Fluminense. Niterói. Disponível em: <http://www2.ic.uff.br/PosGraduacao/Dissertacoes/655.pdf>. Acesso em julho de 2016.

COPE,B.; KALANTZIS,C."Multiliteracies: the beginnings of an Idea.” Literacy learning and the design of social futures. Londres: Routledge. 2000.

GONÇALVES, P. et al. "Comparing and Combining Sentiment Analysis Methods." Disponível em:

<http://homepages.dcc.ufmg.br/ fabricio/download/cosn127goncalves.pdf >. Acesso em junho de 2016.

KRESS, G.; VANLEEUWEN,T. "Multimodal Discourse: the modes and media of contemporary communication". London: Oxford University Press, 2001.Disponível em: <http://neamathisi.com/literacies/chapter-7-literacies-as-multimodal-designsformeaning/kress-and-van-leeuwen-on-multimodality>. Acesso em julho de 2016.

KRESS,G."Multimodality: A social semiotic approach to contemporary communication". London: Routledge. 2010. Parcialmente disponível em:

<https://books.google.com.br/books?id=ihTm_cI58JQC\&pg=PA54\&hl=ptBR\&source =gbs_toc_r\&cad=4\#v=onepage\&q\&f=false $>$. Acesso em julho de 2016.

MALHEIROS,Y.;LIMA,G.“Uma Ferramenta para Analise de Sentimentos em Redes Sociais Utilizando o SenticNet”. Disponível em:

<http://www.lbd.dcc.ufmg.br/colecoes/sbsi/2013/0047.pdf >. Acesso em março de 2017.

MOREIRA,V.S. et al. "Análise de Sentimentos: Comparando o uso de ferramentasea análise humana”. XII Brazilian Symposium on Information Systems, Florianópolis, SC,May17-20,2016. Disponível em:

<http://www.lbd.dcc.ufmg.br/colecoes/sbsi/2016/058.pdf>. Acesso em julho de 2016.

MORENCY, P; et al. "Towards Multimodal Sentiment Analysis: Harvesting Opinions from the Web,” Proc. Int' 1 Conf. Multimodal Interfaces, 2011,pp.169-176.

Disponível em:

$<$ http://ict.usc.edu/pubs/Towards\%20Multimodal\%20Sentiment $\% 20$ Analysis $\% 20$ Harv esting\%20Opinions\%20from\%20The\%20Web.pdf > Acessoemjunhode 2016.

PANG, B.; LEE, L. "Opinion Mining and Sentiment Analysis". Foundations and Trends®inInformationRetrieval:Vol.2:No.1-2,pp1-135.2008.Disponívelem: <http://dx.doi.org/10.1561/1500000011>.Acesso em junho de 2016.

PORIA, S. et al."Towards an Intelligent FrameworkforMultimodalAffectiveData Analysis". Neural Networks(2014). Disponível em: 
<https://dspace.stir.ac.uk/bitstream/1893/21310/1/Neural\%20Networks\%202014.pdf $>$.Acesso em julho de 2016.

PORIA, S. et al."Fusing audio, visual and textual clues for sentiment analysis from multimodalcontent.” Neurocomputing 174, pp.50-59 (2016). Disponível em: <http://sentic.net/multimodal-sentiment-analysis.pdf>. Acesso em maio de 2016.

RAAIJMAKERS, S. et al."Multimodal Subjectivity Analysis of Multiparty Conversation,’Proc.Conf. Empirical Methods in Natural Language Processing, 2008,pp.466-474.

REIS, J. C.S. et al.“Uma Abordagem Multilíngue para Análise de Sentimentos”. 2015. Disponível em: <http://homepages.dcc.ufmg.br/ fabricio/download/brasnam15multi.pdf>. Acesso em junho de 2016.

SANTOS, A. G.; BECKER, K; MOREIRA, V. Um estudo de caso de mineração de emoções em textos multilíngues. BraSNAM - III Brazilian Workshop on Social Networks Analysis and Mining, 2014.

SANTOS, A. G.; BECKER, K; MOREIRA, V. Mineração de emoções em textos multilíngues usando um corpus paralelo. 29 ${ }^{\circ}$ SBBD - SBBD Proceedings, 2014.

SERAFINI, F. "Reading the Visual. An introduction to teaching multimodal literacy". New York. Teachers College Press. 2013

VIAN JR, O. "Gêneros do Discurso, narrativas e avaliação nas mudanças sociais: a análise de discurso positiva". In: Cadernos de Linguagem e sociedade.V.11,n.2 (2010). Disponível em: <http://periodicos.unb.br/index.php/les/article/view/2831/2443>. Acesso em julho de 2016. 